\title{
Review of: \\ The expression of ER beta cx in human breast cancer and the relationship to endocrine therapy and survival
}

\author{
G. Skliris, L. Murphy \\ Manitoba Institute of Cell Biology, University of Manitoba, Winnipeg, Canada
}

\author{
Citation of original article: \\ Palmieri C, Lam EW, Mansi J, MacDonald C, Shousha S, Madden P, Omoto Y, Sunters A, \\ Warner M, Gustafsson JA, Coombes RC. The expression of ER beta cx in human breast cancer and the rela- \\ tionship to endocrine therapy and survival. Clinical Cancer Research 2004 Apr 1; 10(7): 2421-8.
}

\begin{abstract}
of the original article
Purpose: Oestrogen receptor (ER) alpha-positive breast cancer is often treated with endocrine therapy using either antioestrogens or aromatase inhibitors. However, $30 \%$ of patients who receive endocrine therapy will derive no benefit from such treatments and may indeed suffer adverse effects. Currently, there are no ways to predict response to such treatments. ER beta cx, a variant of ER beta, has a dominant-negative effect over ER alpha, and its expression thought to modulate response to endocrine treatment may represent a predictor of response to endocrine therapy.

Experimental design: We investigated the expression of the ER beta cx in 82 frozen breast samples ( 8 benign, 1 ductal carcinoma in situ, and 73 malignant) by western blot analysis. The relationship between the expression of ER beta cx variants with prognosis and outcome of endocrine therapy was examined.

Results: There was a statistically significant association between the presence of ER beta cx and the response to endocrine therapy (Fisher's exact test, $P=0.04$ ). We also examined the influence of the ER beta cx status of a tumour on time to progression and death. There was a relationship between the presence of ER beta $\mathrm{cx}$ and survival, with patients whose tumours express ER beta cx having a longer survival rate $(P=0.05)$. Cell-type specificity of expression was assessed by immunohistochemistry on a selection of histological samples.

Conclusions: On the basis of this small group of patients, we conclude that the expression of ER beta cx correlated with favourable response to endocrine therapy. A larger study involving the staining of archival material is currently underway to confirm these preliminary results.
\end{abstract}

\section{Review}

$\mathrm{ER} \alpha$ is a useful but imperfect predictive marker of endocrine therapy response in breast cancer. The

Correspondence to: Leigh Murphy, Manitoba Institute of Cell Biology, University of Manitoba, Winnipeg, Canada R3E 0V9; Tel: +1 204787 4071; Fax: +1 204787 2190; E-mail: Icmurph@cc.umanitoba.ca

Publication date 29/09/04

BCO/295/2004/JC search for additional markers is an extensive research area, and excitement in this regard was heightened with discovery of ER $\beta$. However, its role in breast cancer remains unknown. Data in the literature are contradictory and may be due in part to differential expression of ER $\beta$ variant proteins. One particular variant, $E R \beta c x$, also known as human ER $\beta 2$ was suggested as a dominant-negative inhibitor of $E R \alpha$ [1], and has been identified at both the RNA and protein levels in breast cancer. Due to alternative splicing, 
$E R \beta c x / 2$ mRNA encodes a non-ligand binding ER $\beta$ variant protein identical to the long form of ER $\beta 1$ up to amino acid 468 followed by 27 amino acids that are distinct from those in ER $\beta 1$. The current paper uses western blotting and immunohistochemistry $(\mathrm{IHC})$ to investigate expression of ERßcx in normal and neoplastic human breast tissue, and correlates expression with clinical outcome and response to endocrine therapy. Western blotting and IHC used sheep antibodies raised to a peptide specific for $E R \beta c x$. ER $\beta c x$ positivity was defined as presence or absence of an appropriately sized immunoreactive protein by western blotting (although no examples of samples classified as negative on western blotting were indicated). In a subset of tumours $(n=23)$ western blotting data were correlated with IHC. IHC was scored by addition of staining intensity score and proportion of cells staining. No indication of staining location (nuclear or cytoplasmic or both) was given. Using $\mathrm{IHC}$ scores $>2$ as ER $\beta \mathrm{cx}$ positive, there was $>80 \%$ correlation of $\mathrm{IHC}$ and western blot results.

The key findings are:

- ERßcx status as defined categorically (+/-) by western blotting in invasive breast cancers $(n=73)$ was not significantly associated with any clinicopathological features of the primary tumours.

- Positive ER $\beta c x$ status of primary tumours defined categorically as above was not associated with time to progression but was significantly associated with a longer survival rate than those patients whose tumours were ERßcx negative.

- In a small group of patients $(n=23)$ who received endocrine therapy, positive ER $\beta c x$ status again defined categorically as above was significantly associated with a better response to endocrine therapy.

The authors suggest, that although the mechanism is unclear, these preliminary data support a link between ER $\beta c x$ expression and response to endocrine therapy, but a larger study is required to confirm the observation. To date there are three published studies, including the current one, where a specific antibody to $E R \beta c x / 2$ was used to investigate protein expression in breast tissue in vivo. Two of these studies [2,3] used the same antibody (raised in sheep to ER $\beta c x$ C-terminal specific peptide) and the third study [4] used a different antibody (raised in rabbits to the same ER $\beta c x$ C-terminal peptide). Although different criteria for assigning ERßcx positivity and negativity were used similar frequencies of ERßcx positivity (48\% [2], 52\% [3], 54\% [4]) in invasive human breast cancers were obtained. As well in the two studies $[2,4]$ that compared frequency of ER $\beta \mathrm{cx}$ positivity in normal/benign breast tissue, both identified a significantly reduced frequency of ERßcx positivity in normal/benign tissues compared to tumours. These results correlate with data obtained at the RNA level $[4,5]$. Despite these similarities, in the two studies $[2,3]$ where ER $\beta c x$ positivity and endocrine therapy response were assessed, opposite conclusions were drawn. However, conclusions from these studies must be underscored with caution since there are distinct differences between them, and both suffer from extremely small sample sizes of $n=23$ [2] and $n=18$ [3], respectively. The authors argue that differences observed between the two studies, were due (a) to the different techniques employed (immunoblotting vs. $\mathrm{IHC}$ ) although similar frequencies of ERßcx positivity and negativity in the populations studied could argue against this being a significant issue; and (b) the different populations studied. In study [3] the population was newly diagnosed patients treated neoadjuvantly for 3 months before tumour removal and response of the primary tumour to treatment was assessed, compared to the current study [2] where surgery had generally been undertaken and the patients identified to have locally advanced or metastatic disease. In addition the type of therapy (neoadjuvant vs. neoadjuvant and palliative; numbers per group not given) and length of follow-up (3 months vs. > 3 months to years; median times of follow-up not given) were quite different between the two studies.

The findings of the current paper are potentially important. But clearly, larger patient cohorts and standardized methodologies need to be undertaken before robust conclusions can be drawn. If these data are confirmed in a larger study, then a new marker, ERßcx, for determining endocrine therapy response would be established, and its value alone or together with other previously established markers should be evaluated using appropriate clinical trials material together with standardized methodologies. This would in turn support extensive investigation of functional roles of ERßcx in mediating sensitivity to endocrine therapy to identify possible novel targets/approaches for therapy.

\section{References}

1. Ogawa S, Inoue S, Watanabe T, et al. Molecular cloning and characterization of human estrogen receptor $\beta c x$ : a potential inhibitor of estrogen action in human. Nucleic Acids Res 1998; 26: 3505-3512.

2. Palmieri C, Lam EW, Mansi J, et al. The expression of ER beta $\mathrm{cx}$ in human breast cancer and the relationship to endocrine therapy and survival. Clin Cancer Res 2004; 10: 2421-2428.

3. Saji S, Omoto Y, Shimizu C, et al. Expression of estrogen receptor (ER) (beta)cx protein in ER(alpha)-positive breast cancer: specific correlation with progesterone receptor. Cancer Res 2002; 62: 4849-4853. 
4. Omoto $\mathrm{Y}$, Kobayashi S, Inoue S, et al. Evaluation of oestrogen receptor beta wild-type and variant protein expression, and relationship with clinicopathological factors in breast cancers. Eur J Cancer 2002; 38: 380-386.
5. Leygue E, Dotzlaw $\mathrm{H}$, Watson $\mathrm{PH}$, et al. Expression of estrogen receptor beta1, beta2, and beta5 messenger RNAs in human breast tissue. Cancer Res 1999; 59: 1175-1179. 